\title{
Evaluation of BODE index and its relationship with systemic inflammation mediated by proinflammatory biomarkers in patients with COPD
}

\author{
Naushad Ahmad Khan ${ }^{1,2}$ \\ Mradul Kumar Daga' \\ Istaq Ahmad ${ }^{2}$ \\ Govind Mawari' \\ Suman Kumar ${ }^{3}$ \\ Naresh Kumar' \\ Syed Akhter Husain ${ }^{2}$ \\ 'Department of Medicine, Maulana \\ Azad Medical College, ${ }^{2}$ Department \\ of Biosciences, Jamia Millia Islamia, \\ ${ }^{3}$ Department of Microbiology, \\ Maulana Azad Medical College, \\ New Delhi, India
}

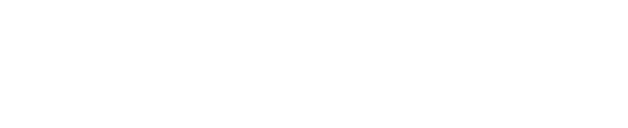

Introduction: BODE index, a multidimensional grading system which is based on Body mass index, airway Obstruction, Dyspnea scale, and Exercise capacity, has been increasingly used for the evaluation of chronic obstructive pulmonary disease (COPD). Many of the systemic manifestations of COPD are shown to be mediated by elevated levels of proinflammatory biomarkers. Objective: We aimed to investigate the relationship between the BODE index, its components, disease severity, and proinflammatory biomarkers like C-reactive protein (CRP), tumor necrosis factor (TNF)- $\alpha$, and interleukin (IL)-6.

Materials and methods: A cross-sectional study which included 290 clinically stable COPD patients and 80 smoker controls was conducted. Medical history, body mass index, pulmonary function tests, 6-minute walking test, and modified scale of Medical Research Council dyspnea scale were evaluated. BODE scores were determined. Systemic inflammation was evaluated with the measurement of CRP, TNF- $\alpha$, and IL- 6 in the serum samples of all studied subjects. The correlation between inflammatory biomarkers and BODE index was assessed in COPD patients. Results: We found a significant relationship between COPD stages and BODE index. Our analysis showed significant association between systemic biomarkers and components of the BODE index. Both TNF- $\alpha$ and CRP levels exhibited weak but significant correlation with BODE index. Serum IL-6 concentrations exhibited significant correlation with 6-minute walking test, modified scale of Medical Research Council, and BODE index ( $r=0.201, P=0.004 ; r=0.068$, $P=0.001$; and $r=0.530, P=0.001$, respectively). Also, an inverse and significant correlation was observed between BODE index and FEV1 $(r=0.567, P=0.001)$. IL-6 exhibited a highly significant and inverse correlation with FEV1 $(r=-0.580, P=0.001)$.

Conclusion: BODE index should be considered for evaluating patients with COPD. Also, IL-6 seems to be a potential biomarker that may enable determination of the severity and prediction of the course of the disease.

Keywords: BODE index, chronic obstructive pulmonary disease, systemic inflammation, biomarkers

\section{Introduction}

Chronic obstructive pulmonary disease (COPD) is a disorder characterized by progressive development of airflow limitation and an enhanced chronic inflammatory response in the airways, ${ }^{1}$ and is predicted to become the third most frequent cause of death in the world by $2030 .^{2}$

The major manifestation of airflow obstruction in COPD is the reduction in forced expiratory volume in 1 second (FEV1). ${ }^{3}$ However, according to the European 
Respiratory Society and the American Thoracic Society (ATS), the measurement of FEV1 alone does not represent the complex clinical consequences of COPD and additional parameters should be assessed and evaluated. ${ }^{4}$ Recent developments in the past decades have led to better understanding of the systemic nature of COPD, which has given rise to the multidimensional classification system that systematically predicts the degree of mortality in individuals with COPD. The BODE index, which is based on Body mass index (BMI), airway Obstruction, Dyspnea scale, and Exercise capacity, includes both symptoms and physiological measurements, and now it is being considered as better indicator than FEV1 for predicting mortality and severity of COPD. ${ }^{5}$ According to the Global initiative for chronic Obstructive Lung Disease (GOLD), the BODE index gives more comprehensive information in predicting mortality from any cause as well as respiratory causes than FEV1-based staging system. ${ }^{5,6}$

It is now recognized that COPD is characterized by lowgrade chronic systemic inflammation, and therefore, it is an important component of COPD. ${ }^{7}$ The role of inflammatory cytokines has also been widely investigated in the natural history of COPD. The inflammation in the respiratory tract of COPD patients seems to be an amplification of the normal inflammatory response of the respiratory tract to chronic irritants. Inflammatory cells like macrophages, neutrophils, and lymphocytes release inflammatory mediators which interact with structural cells in the airways and the lung parenchyma. Various inflammatory mediators, such as cytokines, chemokines, growth factors, and reactive oxygen species, are found to be increased in COPD patients. ${ }^{8} \mathrm{C}$-reactive protein (CRP) levels have been shown to be elevated in the serum of even stable COPD patients and found to be associated with disease severity, quality of life, exercise capacity, response to treatment, and mortality. ${ }^{9}$

Raised levels of proinflammatory cytokines such as interleukin (IL)-6 have been reported in the circulation of stable COPD patients ${ }^{7,10}$ and shown to be associated with impaired functional capacity, ${ }^{11}$ reduced daily physical activity, ${ }^{12}$ and decreased health status. ${ }^{9}{ }^{10}$ Tumor necrosis factor- $\alpha$ (TNF- $\alpha$ ) has been reported to play an important role in muscle wasting and weight loss occurring in COPD patients. ${ }^{13}$ Several studies have shown elevated levels of TNF- $\alpha$ and its receptors in the circulation of COPD patients. ${ }^{14,15}$ It is not clear whether these cytokines are simply markers of the inflammatory process in COPD or differences in inflammation are related to different phenotypes of the disease. ${ }^{16}$ The inflammatory processes that underlie COPD are probably mediated by a multitude of cytokines of proinflammatory cascade. Systemic inflammatory biomarkers may offer new strategies for the diagnosis and may be valuable in assessing the prognosis of patients with COPD.

There are only a few studies that have investigated the relationship between the clinical characteristics of this disease severity (measured by the BODE index) and the systemic inflammation mediated by proinflammatory cytokines, despite there being increasing scientific evidences. However, no study has reported the significance of the differences in the levels of multiple proinflammatory cytokines among different scores and quartiles of the BODE index in patients with COPD in the Indian population.

In the present study, we hypothesize that the BODE index would be a better predictor of systemic inflammation and health status in COPD patients than FEV1 alone, and would present significant associations with biomarkers of systemic inflammation. We also planned to investigate the correlation between the components of BODE index and the BODE index itself with systemic inflammatory biomarkers in patients with stable COPD.

\section{Materials and methods Ethics statement}

The study was approved by the Institutional Ethics Committee review board of Maulana Azad Medical College and Associated Lok Nayak Hospital, New Delhi, India. All the subjects received written information and provided written informed consent prior to participation in the study.

\section{Patient selection}

Two hundred and ninety stable COPD patients diagnosed according to the GOLD guidelines in the pulmonary outpatient clinic of Maulana Azad Medical College and associated Lok Nayak Hospital were included in the study between November 2011 and December 2013. Eighty smokers (65 males and 15 females) from the general population were recruited as the control group. The inclusion criterion was: COPD patients in stable conditions (no exacerbations due to any reason in the last 4 weeks). Stable COPD patients had been receiving inhaled bronchodilator therapy in the form of long-acting $\beta 2$-agonists and/or anticholinergic agents. Severe/very severe COPD patients were on inhaled corticosteroids as well. COPD was defined as FEV1/forced vital capacity (FVC) ratio of less than $70 \%$ at 20 minutes after salbutamol administration. The exclusion criteria were: presence of a respiratory disorder other than COPD or other inflammatory diseases (inflammatory bowel disease, vasculitis), presence of atopy, and history of myocardial infarction 
in the last 6 months, decompensated cardiovascular disease, and walking disability.

\section{Smoker controls}

Male/female subjects aged 35-75 years without any significant disease as determined by history and physical examination and current and ex-smokers with a smoking history of $\geq 10$ pack-years with normal pulmonary function were enrolled as controls.

Demographic features and medical history of the subjects were recorded. Weight, height, and dyspnea severity were measured, and the 6-minute walking test (6MWT) and the pulmonary function tests (PFTs) were performed. Measurement of serum levels of inflammatory biomarkers (CRP, TNF- $\alpha$, IL-6) was also performed.

\section{PFTs (spirometry)}

PFTs were done for stable COPD patients in the outpatient clinic. FEV1 and FVC were measured with a calibrated spirometer (Spirolab III, MIR, Via Del Maggiolino, Roma, Italy; a portable pulmonary function apparatus). Patients with FEV1 $\geq 80 \%$ of the predicted value were considered mild, those with $50 \% \leq \mathrm{FEV} 1<80 \%$ of the predicted value were considered moderate, those with $30 \% \leq \mathrm{FEV} 1<50 \%$ of the predicted value were considered severe, and patients with FEV1 $<30 \%$ of the predicted value were considered very severe. The best value from three consecutive tests was accepted. FEV1, FVC, and FEV1/FVC were measured according to ATS criteria. COPD staging was done according to GOLD $2011 .^{6}$

\section{Anthropometric, body composition, exercise capacity, and dyspnea assessments}

During the clinical visit, the information about demographics and a detailed medical history were obtained from the patients. Height was measured in centimeters and weight in kilograms by a calibrated scale with the subjects not wearing their shoes. The BMI was calculated as weight (in kilograms)/height ${ }^{2}$ (in meters). Functional exercise capacity was measured with the 6MWT in accordance with the ATS recommendations. ${ }^{17}$ The $6 \mathrm{MWT}$ was performed in a level, covered hospital corridor of approximately $50 \mathrm{~m}$ in length. Three tests were performed and the test with the maximum 6-minute walking distance (6MWD) was considered for analysis. Each patient received standard instructions and encouragement during the test. The magnitude of dyspnea was assessed using the modified scale of Medical Research Council (mMRC). ${ }^{18}$ Patients were asked about their perceived breathlessness and were then classified into the mMRC five dyspnea grades ( 0 minimum to 4 maximum).

\section{BODE index}

The BODE index was calculated for each participant based on the combination of four variables, with the following scores: a measure of body composition (BMI) from 0 to 1 point; a measure of the intensity of airflow obstruction (FEV1\% predicted postbronchodilator): from 0 to 3 points; a measure of subjective sensation of dyspnea (MRC scale): from 0 to 3 points; and a measure of exercise capacity (walked distance in the 6MWT): from 0 to 3 points. The final score of the BODE index ranges from 0 to 10 points; higher the index value, the worse is the patient's condition. The participants were divided into four quartiles for the analysis according to their BODE index score, as previously described by Celli et al. ${ }^{5}$ Quartile I is a score of 0-2 points, quartile II 3-4 points, quartile III 5-6 points, and quartile IV is a score of $7-10$ points.

\section{Measurement of inflammatory biomarkers (CRP,TNF- $\alpha$, IL-6)}

Five milliliters of venous blood sample was collected and centrifuged at $1,000 \times g$ for 15 minutes. The serum samples were separated, aliquoted, and preserved at $-80^{\circ} \mathrm{C}$ until further analysis. Serum CRP levels were measured with the original reactive analyzers (Unicel DxC 800 Synchron Clinical Systems; Beckman Coulter Inc., Galway, Ireland). Serum TNF- $\alpha$, IL-6 (Thermo Fisher Scientific, Waltham, MA, USA), and CRP (Thermo Fisher Scientific) were measured according to the manufacturer's instructions with the enzyme-linked immunosorbent assay method. The intra-assay coefficient of variation $(\% \mathrm{CV})$ values for TNF- $\alpha$ kit were $4.2 \%$ for $87.5 \mathrm{pg} / \mathrm{mL}$ and $4.5 \%$ for $369 \mathrm{pg} / \mathrm{mL}$, while the interassay coefficients of variation were $5.2 \%$ for $92.8 \mathrm{pg} / \mathrm{mL}$ and $5.0 \%$ for $384 \mathrm{pg} / \mathrm{mL}$. The lowest limit of detection for TNF- $\alpha$ kit was $<2 \mathrm{pg} / \mathrm{mL}$. The intra-assay precision value for IL-6 kit was $\leq 9.4 \%$, while the interassay precision was $\leq 8.6 \%$. The lowest measurement level for IL- 8 kit was $<1.0 \mathrm{pg} / \mathrm{mL}$. The intra-assay coefficient of variation $(\% \mathrm{CV})$ values for CRP kit were $6.02 \%$ for $51.01 \mathrm{pg} / \mathrm{mL}$ and $9.82 \%$ for $205.01 \mathrm{pg} / \mathrm{mL}$, while the interassay coefficients of variation were $9.98 \%$ for $56.98 \mathrm{pg} / \mathrm{mL}$ and $9.82 \%$ for $229.33 \mathrm{pg} / \mathrm{mL}$.

\section{Statistical analysis}

Data were analyzed by the Statistical Package for the Social Sciences 20.0 package program (IBM Corporation, Armonk, NY, USA). The normality of data distribution was analyzed by the Kolmogorov-Smirnov test. The results are presented as mean \pm standard deviation (SD), median (range), or proportions (percentage), depending upon their distribution and the measurement scale. Baseline 
differences between different studied groups were determined with unpaired student's $t$-test and one way ANOVA. In general, the Kruskal-Wallis test with Dunn's post-test was used to compare the variables of the participants in different quartiles of the BODE index, the Mann-Whitney test to evaluate the differences in the biomarkers' levels between the groups "quartiles I-II" and "quartiles III-IV", and the Spearman correlation coefficient was used to correlate the BODE index and the studied biomarker variables. Statistical significance was set at $P$-values $<0.05$ (two-sided) for all analyses.

\section{Results}

\section{Demographics and clinical characteristics of the study subjects}

Table 1 presents the main demographic and clinical characteristics of all participants at recruitment. On average, COPD patients had moderate to severe airflow limitation and as expected, they complained of more symptoms than controls. A total of 290 COPD patients (254 males and 36 females) with a mean age $58.3 \pm 16.1$ years were recruited in the study. Also, 80 smokers (65 males and 15 females) were recruited in the study from the general population (mean age $47.2 \pm 11.0$ years). There was a higher proportion of males in patients and controls compared with the healthy smokers.
COPD patients had more pack-years of smoking (38.6 \pm 17.4 vs 17.4 \pm 9.5 years) and clear functional exercise intolerance $(6 \mathrm{MWD}=329 \pm 122$ vs $510 \pm 133 \mathrm{~m})$. As expected, COPD patients had significant moderate to severe airflow obstruction $(\mathrm{FEV} 1=54.3 \pm 12.8)$, compared to healthy smokers $($ FEV1 $=102.8 \pm 16.3)$ who had normal spirometry $(P<0.001)$. COPD patients and smoker controls did not differ in BMI $(P=0.42)$. BODE index score and mMRC score were found to be significantly higher in COPD patients compared to smoker controls $(P<0.001)$ (Table 1$)$. On crude comparison, we found that COPD patients had higher concentrations of serum inflammatory biomarkers (TNF- $\alpha$, IL-6, and CRP) than the controls and they differed significantly $(P<0.001)$.

\section{Relationship between serum biomarkers and severity parameters stratified by GOLD}

Table 2 shows the characteristics of the COPD patients stratified according to different stages of GOLD. The patients represented three stages of COPD severity. One hundred and forty-seven COPD patients were in GOLD II stage, 104 in GOLD III stage, and 39 were in GOLD IV stage. Age, BMI, pack-years, and pulmonary function parameters differed significantly with increasing GOLD stage. As expected, exercise capacity measured by 6MWT decreased significantly with

Table I Baseline characteristics of the COPD patients and smoker controls

\begin{tabular}{|c|c|c|c|}
\hline Characteristics & COPD subjects $(n=290)$ & Smoker controls $(n=80)$ & $P$-value \\
\hline Age (years) & $58.3 \pm 16.1$ & $47.2 \pm 11.0$ & $<0.001$ \\
\hline Male/female & $254 / 36$ & $65 / 15$ & - \\
\hline Smoking (years) & 38.7 & 16.4 & $<0.001$ \\
\hline Pack-years & $38.6 \pm 17.4$ & $17.4 \pm 9.5$ & $<0.001$ \\
\hline BMI $\left(\mathrm{kg} / \mathrm{m}^{2}\right)$ & $22.4 \pm 6.2$ & $22.8 \pm 4.6$ & 0.42 \\
\hline FEVI (\% predicted) & $54.3 \pm 12.8$ & $102.8 \pm 16.3$ & $<0.001$ \\
\hline FEVI/FVC (\%) & $45.8 \pm 11.6$ & $79.2 \pm 5.4$ & $<0.001$ \\
\hline FEVI reversibility (\%) & $10.7 \pm 13.7$ & $4.5 \pm 5.8$ & $<0.001$ \\
\hline FVC (\%) & $86.4 \pm 18.6$ & $110.4 \pm 13.5$ & $<0.001$ \\
\hline 6-minute walking distance $(\mathrm{m})$ & $329 \pm 122$ & $510 \pm 133$ & $<0.001$ \\
\hline BODE index score & $5.20 \pm 3.9$ & $0(0-1)$ & $<0.001$ \\
\hline Quartile I (0-2) & $193(66.5 \%)$ & $80(100 \%)$ & - \\
\hline Quartile $2(3-4)$ & $4 \mid(14.1 \%)$ & 0 & $<0.001$ \\
\hline Quartile 3 (5-6) & $36(12.4 \%)$ & 0 & - \\
\hline Quartile $4(7-10)$ & $20(6.8 \%)$ & 0 & - \\
\hline mMRC score & $1.8 \pm 1.3$ & $0.8 \pm 0.2$ & $<0.001$ \\
\hline \multicolumn{4}{|l|}{ Inflammatory variables } \\
\hline TNF- $\alpha(p g / m L)$ & $4.09 \pm 1.38$ & $3.76 \pm 1.39$ & $<0.001$ \\
\hline IL-6 (pg/mL) & $1.56 \pm 0.98$ & $1.16 \pm 0.73$ & $<0.001$ \\
\hline CRP (pg/mL) & $4.52 \pm 2.26$ & $2.88 \pm 1.12$ & $<0.001$ \\
\hline
\end{tabular}

Note: Values are expressed as mean (standard deviation) or as percentage. P-value's were calculated via unpaired Student's $t$-test.

Abbreviations: BMI, body mass index; BODE, BMI (B), airflow obstruction (O) as measured by the postbronchodilator FEVI (percentage of predicted value), dyspnea (D) assessed by the mMRC score, and exercise tolerance (E) measured by 6-minute walking distance; COPD, chronic obstructive pulmonary disease; CRP, C-reactive protein; FEVI, forced expiratory volume in I second; FVC, forced vital capacity; IL-6, interleukin 6; mMRC, modified Medical Research Council scale; TNF- $\alpha$, tumor necrosis factor alpha. 


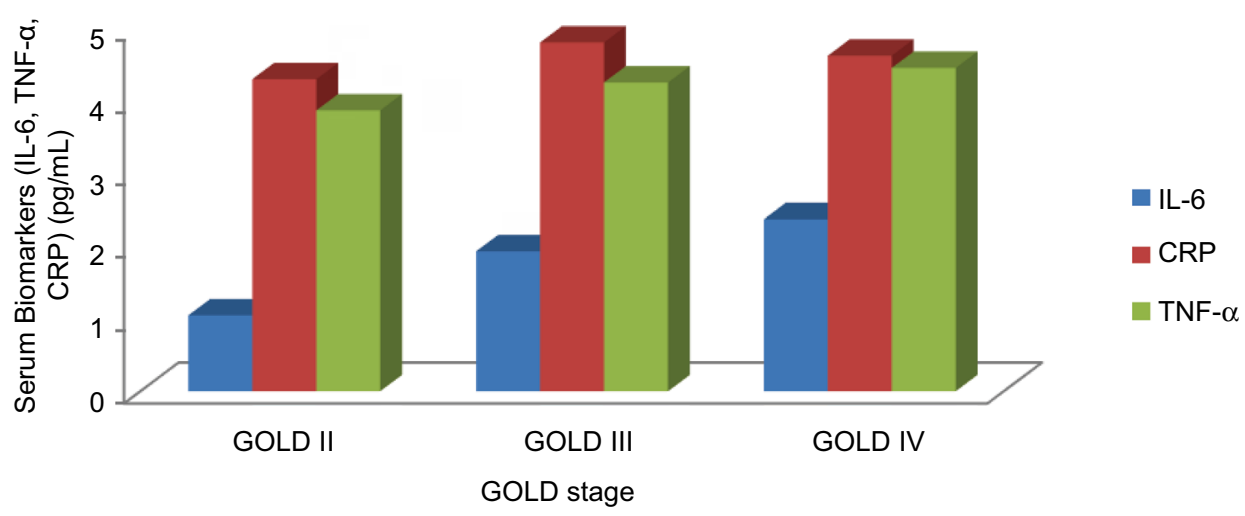

Figure I Distribution of inflammatory biomarkers (IL-6, CRP, and TNF- $\alpha$ ) stratified by GOLD stage in COPD patients.

Abbreviations: COPD, chronic obstructive pulmonary disease; CRP, C-reactive protein; GOLD, Global Initiative for Chronic Obstructive Lung Disease; IL-6, interleukin 6; TNF- $\alpha$, tumor necrosis factor alpha.

Table 2 Baseline characteristics of COPD according to different stages of GOLD

\begin{tabular}{|c|c|c|c|c|}
\hline Parameter & GOLD II $(n=\mid 47)$ & GOLD III $(n=104)$ & GOLD IV $(n=39)$ & $P$-value \\
\hline Age & $53.08 \pm 9.12$ & $56.22 \pm 10.45$ & $58.28 \pm 10.05$ & 0.005 \\
\hline BMI & $20.74 \pm 2.27$ & $21.37 \pm 2.70$ & $21.69 \pm 2.30$ & 0.0002 \\
\hline Pack-years & $24.80 \pm 8.45$ & $25.3 I \pm 8.75$ & $30.10 \pm 10.68$ & $<0.001$ \\
\hline FEVI (\%) & $62.15 \pm 6.90$ & $41.03 \pm 6.23$ & $27.25 \pm 2.57$ & $<0.001$ \\
\hline FVC & $71.91 \pm 9.84$ & $56.06 \pm 8.29$ & $42.19 \pm 6.83$ & $<0.001$ \\
\hline FEVI/FVC\% & $60.68 \pm 4.7$ & $49.63 \pm 7.05$ & $38.50 \pm 5.43$ & $<0.001$ \\
\hline 6MWT & $235.35 \pm 59.59$ & $221.94 \pm 63.59$ & $190.47 \pm 47.83$ & $<0.001$ \\
\hline $\mathrm{mMRC}$ score & $1.7 \pm 1.2$ & $2.1 \pm 1.1$ & $2.5 \pm 1.4$ & $<0.001$ \\
\hline TNF- $\alpha$ (pg/mL) & $4.14 \pm 1.33$ & $4.25 \pm 1.31$ & $4.45 \pm 1.57$ & 0.005 \\
\hline CRP $(\mathrm{pg} / \mathrm{mL})$ & $4.29 \pm 1.21$ & $4.80 \pm 1.25$ & $4.62 \pm 1.32$ & 0.04 \\
\hline IL-6 (pg/mL) & $1.04 \pm 0.50$ & $1.92 \pm 1.00$ & $2.36 \pm 1.14$ & 0.002 \\
\hline BODE index & $2.09 \pm 1.59$ & $5.96 \pm 1.34$ & $7.24 \pm 1.62$ & $<0.001$ \\
\hline
\end{tabular}

Note: Values are expressed as mean (standard deviation) or as percentage. P-value's were calculated via ANOVA.

Abbreviations: 6MWT, 6-minute walking test; BMI, body mass index; BODE, BMI (B), airflow obstruction (O) as measured by the postbronchodilator FEVI (percentage of predicted value), dyspnea (D) assessed by the mMRC score, and exercise tolerance (E) measured by 6-minute walking distance; COPD, chronic obstructive pulmonary disease; CRP, C-reactive protein; FEVI, forced expiratory volume in I second; FVC, forced vital capacity; GOLD, Global Initiative for Chronic Obstructive Lung Disease; IL-6, interleukin 6; mMRC, modified Medical Research Council scale; TNF- $\alpha$, tumor necrosis factor alpha.

increasing disease severity. All the studied biomarker values changed significantly and increased with worsened disease severity (Figure 1). Interestingly, among the three biomarkers studied, IL-6 was found to be the most consistent for the severity as assessed by GOLD $(P=0.002)$. BODE index scores also increased significantly with increasing disease severity $(P<0.001)$ (Table 2).

\section{Relationship between proinflammatory biomarkers and the clinical parameters of COPD}

In COPD subjects, serum concentrations of inflammatory biomarkers were assessed according to different quartiles of BODE index scores. In general, all the studied biomarker values changed significantly with increasing disease severity (quartiles I-IV) and the differences were found to be concordant with the severity assessed by BODE index scores. The mMRC score and airflow limitation increased significantly in proportion to different quartiles of BODE index (I-IV) and 6MWD decreased significantly from quartile I to IV of BODE index (Figure 2). Age, BMI, and pack-years also differed significantly with worsened disease severity as assessed by BODE index scores. Of the three biomarkers studied, IL-6 was found to be the most consistent biomarker that differed significantly with increased disease severity as stratified by low quartile to high quartile of BODE index $(P<0.001)$ (Table 3$)$.

\section{Associations of the biomarker panel with FEVI, BMI, mMRC, 6MWD, and BODE index}

In COPD patients, the relationships between inflammatory biomarker concentrations, BMI, 6MWT, mMRC, and BODE index were evaluated (Figure 3). The relationship of individual biomarkers with BMI, mMRC, 6MWD, and BODE index is shown in Table 4. A weak and negative correlation 
was noted between TNF- $\alpha$ and 6MWT ( $r=-0.126, P=0.073)$. TNF- $\alpha$ correlated significantly with dyspnea intensity, as assessed by mMRC $(r=0.214, P=0.002)$ and the BODE index ( $r=0.251, P=<0.001)$. CRP levels failed to show any significant correlation with BMI, 6MWT, and mMRC. However, serum IL-6 concentrations exhibited significant correlations with 6MWT, mMRC, and BODE index $(r=0.201, P=0.004$; $r=0.068, P=0.001$; and $r=0.530, P=0.001$, respectively). In this study, we found an inverse and significant correlation between BODE index and FEV1 $(r=0.567, P=0.001)$. None of the biomarkers studied showed significant correlation with BMI. The serum concentrations of the inflammatory

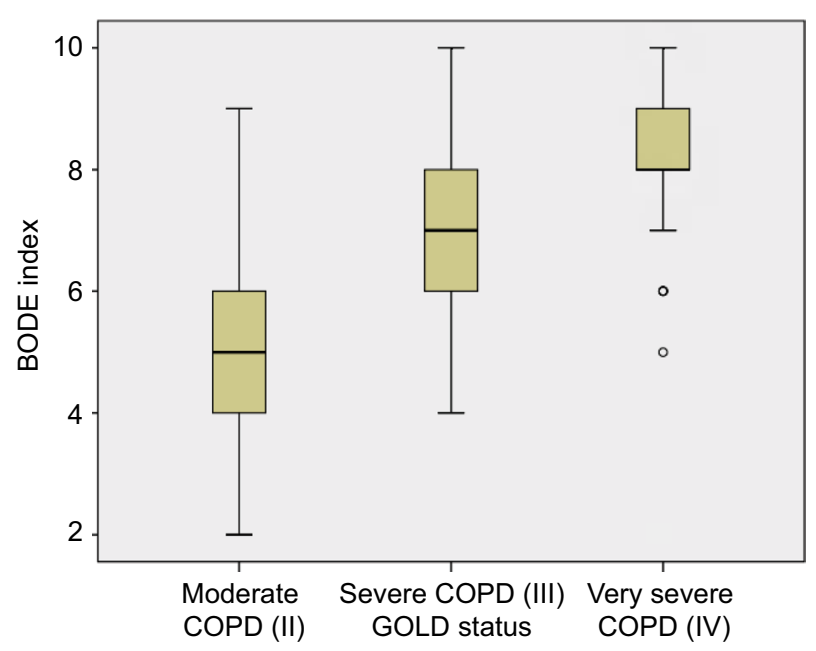

Figure 2 Relationship between BODE index score and GOLD stage in COPD patients.

Abbreviations: BODE, body mass index (B), airflow obstruction $(\mathrm{O})$ as measured by the postbronchodilator forced expiratory volume in I second (percentage of predicted value), dyspnea (D) assessed by the modified Medical Research Council scale score, and exercise tolerance $(E)$ measured by 6-minute walking distance; COPD, chronic obstructive pulmonary disease; GOLD, Global Initiative for Chronic Obstructive Lung Disease. biomarkers under study showed a weak relationship with the functional characteristics of the COPD patients. FEV1 was found to be weakly and inversely related to TNF- $\alpha(r=-0.183$, $P=0.009)$ and CRP ( $r=-0.210, P=0.003)$. However, IL-6 exhibited a highly significant and inverse correlation with FEV1 ( $r=-0.580, P=0.001$ ) (Table 5; Figure 4). All the markers studied were associated with the physiological indicators of disease, but the strength of the association differed among the biomarkers studied in terms of correlation. Therefore, in our study, IL-6 was found to be the biomarker which showed significant correlation with the maximum functional characteristics in COPD patients.

\section{Discussion}

This study aimed to investigate the correlation between the components of BODE index and the systemic inflammatory biomarkers in patients with stable COPD. We propose that multidimensional assessment of COPD by means of serum biomarkers might be more accurate in predicting the risk and course of disease progression than the assessment made by predictors based on a single biomarker or clinical variables alone. In the current study, the biomarkers panel appears to furnish additional information to various clinical variables and to the BODE index. To our knowledge, our study is the first study from India primarily designed to investigate whether adding a panel of proinflammatory biomarkers could add the significance of BODE index in predicting the severity of the disease.

This cross-sectional study provides three relevant observations. Firstly, it suggests that there exists a proinflammatory state characterized by an increased circulation of many inflammatory cytokines in stable COPD patients. Secondly,

Table 3 Comparison of clinical variables and inflammatory biomarkers according to different quartiles of BODE index in COPD patients

\begin{tabular}{|c|c|c|c|c|}
\hline Parameters & Quartile I & Quartile II & Quartile III-IV & $P$-value \\
\hline Age (years) & $52.06 \pm 7.14$ & $57.32 \pm 8.12$ & $64.16 \pm 11.23$ & 0.004 \\
\hline Pack-years & $23.12 \pm 7.47$ & $27.32 \pm 6.45$ & $31.45 \pm 8.91$ & 0.001 \\
\hline BMI $\left(\mathrm{kg} / \mathrm{m}^{2}\right)$ & $24.3 \pm 3.5$ & $22.4 \pm 4.5$ & $20 \pm 3.2$ & 0.001 \\
\hline FEVI (\% predicted) & $60.8 \pm 13.4$ & $47.4 \pm 13.1$ & $38.8 \pm 7.76$ & $<0.001$ \\
\hline FEVI/FVC (\%) & $52.7 \pm 11.0$ & $44.9 \pm 10.6$ & $36.6 \pm 9.6$ & $<0.001$ \\
\hline FEVI reversibility (\%) & $10.2 \pm 10.5$ & $8.2 \pm 12.4$ & $8.4 \pm \mathrm{II} .4$ & 0.008 \\
\hline mMRC score & $1.1 \pm 0.6$ & $1.8 \pm 0.7$ & $2.2 \pm 0.6$ & $<0.001$ \\
\hline 6MWD (m) & $4 \mid 2 \pm 85.0$ & $316 \pm 65.0$ & $194 \pm 54.0$ & $<0.001$ \\
\hline CRP $(\mathrm{pg} / \mathrm{mL})$ & $3.46 \pm 2.12$ & $3.94 \pm 2.75$ & $4.78 \pm 2.87$ & 0.0002 \\
\hline IL-6 (pg/mL) & $\mathrm{I} .2 \mathrm{I} \pm 0.83$ & $1.97 \pm 1.04$ & $2.88 \pm 1.82$ & $<0.001$ \\
\hline TNF- $\alpha$ (pg/mL) & $3.94 \pm 2.02$ & $4.21 \pm 1.87$ & $4.72 \pm 2.07$ & 0.005 \\
\hline
\end{tabular}

Notes: Values are mean (SD) depending on the distribution. Comparison between groups was done by ANOVA.

Abbreviations: 6MWD, 6-minute walking distance; ANOVA, analysis of variance; BMI, body mass index; BODE, BMI (B), airflow obstruction (O) as measured by the postbronchodilator FEVI (percentage of predicted value), dyspnea (D) assessed by the mMRC score, and exercise tolerance (E) measured by 6MWD; COPD, chronic obstructive pulmonary disease; CRP, C-reactive protein; FEVI, forced expiratory volume in I second; FVC, forced vital capacity; IL-6, interleukin 6; mMRC, modified Medical Research Council scale; SD, standard deviation; TNF- $\alpha$, tumor necrosis factor alpha. 

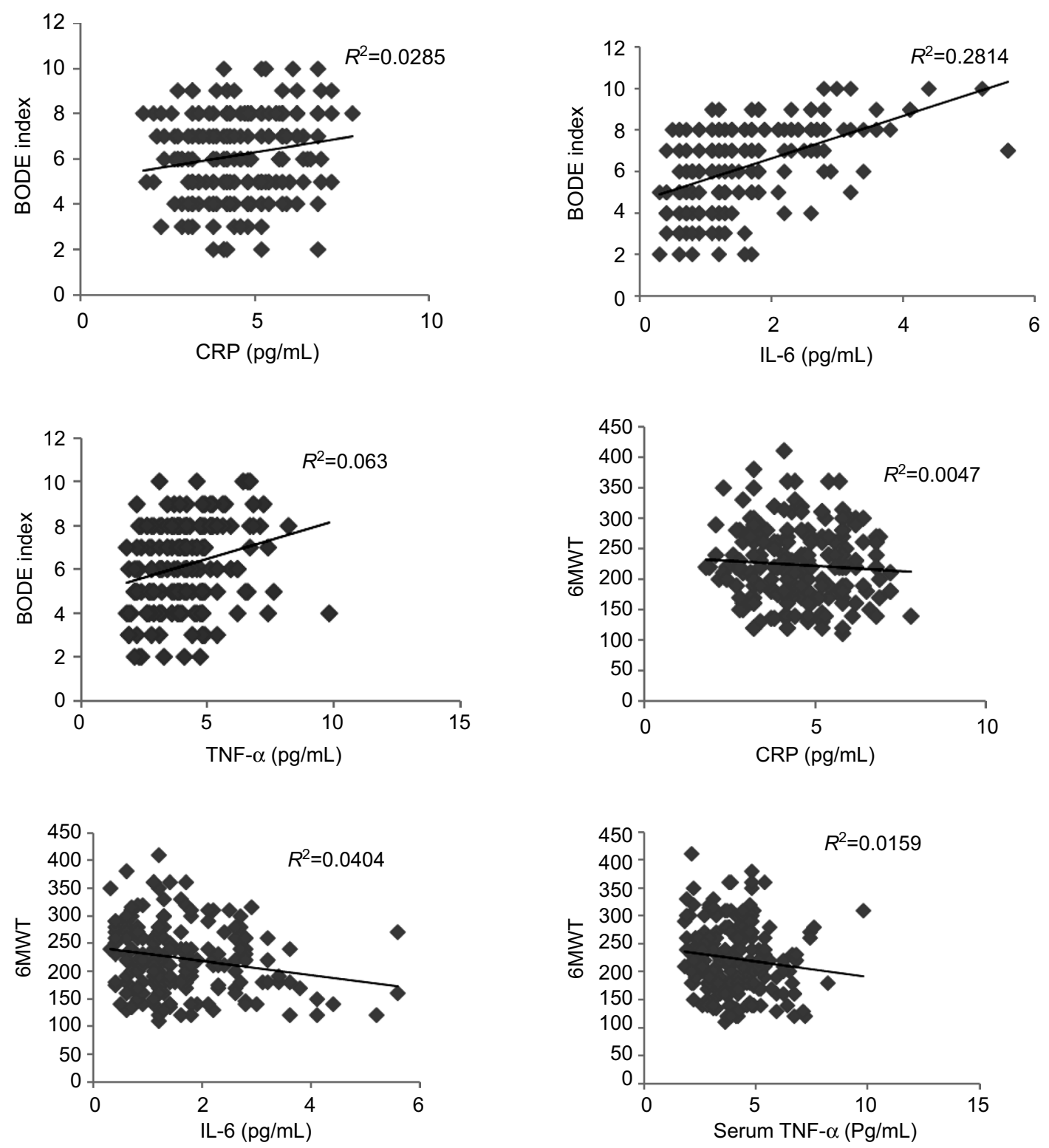

Figure 3 Relationship of serum concentrations of the inflammatory biomarkers studied with the BODE index and 6-minute walk test in COPD patients. Note: $R=$ Pearson's linear bivariate correlation coefficient.

Abbreviations: 6MWT, 6-minute walking test; BODE, body mass index (B), airflow obstruction $(\mathrm{O})$ as measured by the postbronchodilator forced expiratory volume in I second (percentage of predicted value), dyspnea (D) assessed by the modified Medical Research Council scale score, and exercise tolerance (E) measured by 6-minute walking distance; CRP, C-reactive protein; IL, interleukin; TNF- $\alpha$, tumor necrosis factor alpha.

Table 4 Correlations between BMI, 6MWT, mMRC, BODE index, and inflammatory biomarkers in COPD patients

\begin{tabular}{|c|c|c|c|c|}
\hline Biomarkers & BMI & mMRC & 6MWT & BODE index \\
\hline \multirow[t]{2}{*}{ TNF- $\alpha$ (pg/mL) } & $r=-0.030$ & $r=0.214 * *$ & $r=-0.126$ & $r=0.25 \mathrm{I}$ \\
\hline & $P=0.669$ & $P=0.002$ & $P=0.073$ & $P=<0.00 I *$ \\
\hline \multirow[t]{2}{*}{ CRP (pg/mL) } & $r=0.095$ & $r=0.252^{* *}$ & $r=-0.069$ & $r=0.169$ \\
\hline & $P=0.179$ & $P=0.007$ & $P=0.331$ & $P=0.016^{*}$ \\
\hline \multirow[t]{2}{*}{ IL-6 (pg/mL) } & $r=0.104$ & $r=0.068 * *$ & $r=0.201 *$ & $r=0.530^{*}$ \\
\hline & $P=0.139$ & $P=0.00 \mathrm{I}$ & $P=0.004$ & $P=0.00 \mathrm{I}$ \\
\hline \multirow[t]{2}{*}{ BMI $\left(\mathrm{kg} / \mathrm{m}^{2}\right)$} & 1 & $r=0.164^{*}$ & $r=-0.013$ & $r=-0.151 * *$ \\
\hline & & $P=0.002$ & $P=0.08 \mathrm{I}$ & $P=0.032$ \\
\hline
\end{tabular}

Notes: *Correlation is significant at the 0.01 level (two-tailed). ${ }^{* *}$ Correlation is significant at the 0.05 level.

Abbreviations: 6MWT, 6-minute walking test; BMI, body mass index; BODE, BMI (B), airflow obstruction (O) as measured by the postbronchodilator forced expiratory volume in I second (percentage of predicted value), dyspnea (D) assessed by the mMRC score, and exercise tolerance (E) measured by 6-minute walking distance; COPD, chronic obstructive pulmonary disease; CRP, C-reactive protein; IL-6, interleukin 6; mMRC, modified Medical Research Council scale; TNF- $\alpha$, tumor necrosis factor alpha. 
Table 5 Correlation between pulmonary function parameters and inflammatory biomarkers in COPD patients

\begin{tabular}{llll}
\hline Biomarkers & FEVI & FEV I/FVC & FVC \\
\hline TNF- $\alpha(\mathrm{pg} / \mathrm{mL})$ & $r=-0.183^{* *}$ & $r=-0.123$ & $r=-0.209^{* *}$ \\
& $P=0.009$ & $P=0.081$ & $P=0.003$ \\
CRP $(\mathrm{pg} / \mathrm{mL})$ & $r=-0.210^{* *}$ & $r=-0.186^{* *}$ & $r=-0.139^{*}$ \\
& $P=0.003$ & $P=0.008$ & $P=0.048$ \\
$\mathrm{IL}-6(\mathrm{pg} / \mathrm{mL})$ & $r=-0.580^{*}$ & $r=-0.460^{* *}$ & $r=-0.523^{* *}$ \\
& $P=0.001$ & $P=0.001$ & $P=0.001$ \\
\hline
\end{tabular}

Notes: $*$ Correlation is significant at the 0.01 level. ${ }^{*}$ Correlation is significant at the 0.05 level.

Abbreviations: COPD, chronic obstructive pulmonary disease; CRP, C-reactive protein; FEVI, forced expiratory volume in I second; FVC, forced vital capacity; IL-6, interleukin 6; TNF- $\alpha$, tumor necrosis factor alpha.

it shows characterization of the proinflammatory biomarkers in patients with COPD and distinguishes it from that of smokers with normal lung function. Thirdly, it shows that systemic inflammation mediated by IL-6 is a constant feature in all COPD patients that remains persistent and concordant with disease severity as assessed by the GOLD status and BODE index.

The most common clinical component associated with risk prediction in COPD is the severity of airflow obstruction as assessed by FEV1\% predicted. ${ }^{19}$ However, COPD, being a heterogeneous disease, has several extrapulmonary manifestations that are not related to the lung function itself. Hence, it was thought to combine different variables into a multidimensional index that captures the complexity of COPD, such as BODE index, along with systemic biomarkers in order to predict more accurately the risk of disease severity and progression than that predicted by the predictors based on clinical variables alone or any single biomarker.

Several previous studies have demonstrated a weak correlation between PFTs, especially FEV1 and the clinical outcomes including the severity of dyspnea and other symptoms, mortality, quality of life, and the frequency of exacerbations, and BODE index. Ong et $\mathrm{al}^{20}$ found that the BODE index showed a significant but weak relationship between the number of emergency visits and FEV $1 .{ }^{21}$ Another study demonstrated the BODE index to be more significant for determining the severity of COPD exacerbations, in comparison to FEV1. ${ }^{22}$ Similarly, in our study, we found that the BODE index significantly correlated to the FEV1. The above observation $s^{21,22,23}$ highlight the significance of BODE index, as FEV1 does not capture the complexity of the disease alone.

Some of our findings are consistent with those reported in previous cross-sectional studies. Celli et $\mathrm{al}^{5}$ have shown that BMI and BODE index are inversely related. Similar results were observed in our study, which showed a significant decrease in BMI as the BODE index score increased.
BMI plays an important role in defining the phenotypes of COPD patients; low BMI has been shown to be associated with the disease severity and is an independent predictor of mortality in COPD. ${ }^{23,24}$ In the current study, BMI did not correlate with the inflammatory biomarkers studied, suggesting that the loss of body mass may not be mediated by systemic inflammation.

Our study demonstrated higher serum TNF- $\alpha$, CRP, and IL-6 levels in COPD patients than in smoker controls. Several previous studies have also shown higher concentrations of these proinflammatory biomarkers in COPD patients than in healthy smokers and nonsmoker controls. ${ }^{7,10,12}$ Bon et al reported a statistically significant association between the degree of emphysema shown by chest computed tomography and the serum levels of TNF- $\alpha$ and IL- 6 in COPD subjects. ${ }^{25}$ TNF- $\alpha$ is also known to stimulate synthesis of IL- 6 which plays an important role in COPD pathobiology. Hence, our findings are consistent with the findings of previous researchers. Elevated levels of CRP have been associated with impaired exercise capacity, airway obstruction severity, and impaired quality of life in COPD patients. ${ }^{10,12}$ Elevated serum IL-6 levels have been found in COPD patients compared to healthy smokers, ${ }^{12,25,26}$ which is in agreement with our current findings. A previous study had shown the relationship between elevated TNF- $\alpha$ levels and weight loss in COPD.$^{27}$ In our study, we did not find a correlation between TNF- $\alpha$ levels and BMI; however, we observed a significant correlation with BODE index. Our findings are not in line with the findings of Sarioglu et al, ${ }^{28}$ who observed no correlation between TNF- $\alpha$, CRP levels, and BMI. Also they did not report correlation between these biomarkers and BODE index, which is in contrast to our findings. Their failure to establish a significant correlation between BODE index and systemic biomarkers may be attributed to small sample size in their study in comparison to more number of patients included in our study. In the current study, CRP levels failed to show any significant correlation with 6MWT and mMRC. However, serum IL-6 concentrations exhibited significant correlation with 6MWT, mMRC, and BODE index.

IL-6 is a proinflammatory biomarker which plays a key role in COPD pathobiology. It is released by bronchial epithelium, and CD8+ and CD4+ T-cells. ${ }^{29}$ Monocytes of COPD are thought to be involved in overreacting and, thereby, releasing more IL-6 than in healthy subjects. ${ }^{30}$ In the current study, IL-6 concentrations were found to be significantly elevated in COPD patients than in healthy smokers, indicating its potential involvement in the inflammatory process of COPD patients. In agreement with our finding, Pinto-Plata 

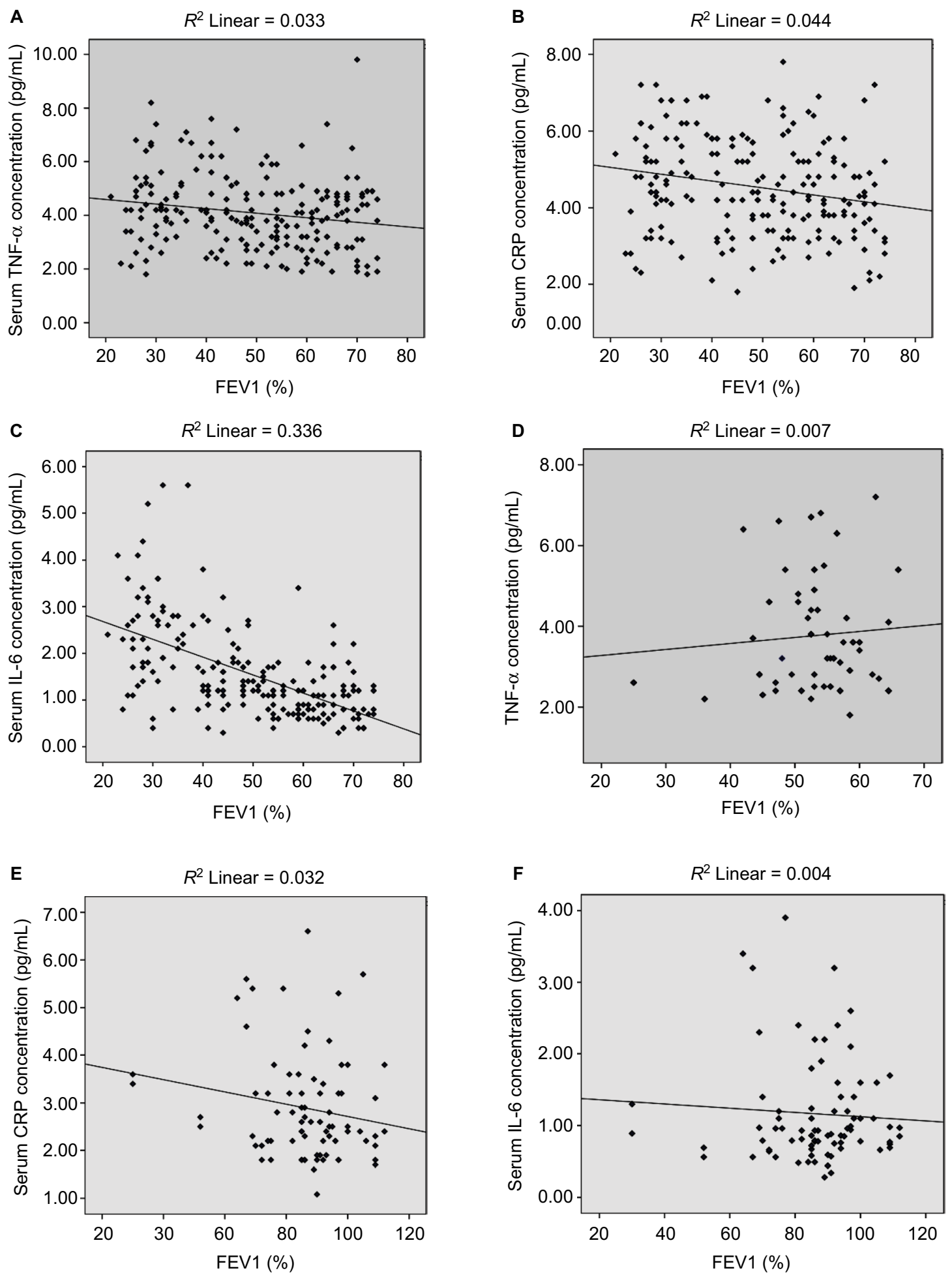

Figure 4 Correlation between serum biomarkers and FEVI\% in COPD patients and smoker controls (A, B, C: COPD patients; D, E, F: smoker controls).

Abbreviations: COPD, chronic obstructive pulmonary disease; CRP, C-reactive protein; FEVI, forced expiratory volume in I second; IL-6, interleukin 6; TNF- $\alpha$, tumor necrosis factor alpha.

et $\mathrm{al}^{31}$ have also shown previously that the serum levels of IL- 6 were elevated in patients with advanced stage of COPD. ${ }^{32}$ In our study, serum IL- 6 was a better marker as compared to TNF- $\alpha$ CRP with respect to BODE index as it showed significant correlation with the maximum functional components of BODE index. (BMI, 6MWT, FEV1, dyspnea). Our findings are in contrast with the results of Gaki et al, ${ }^{33}$ who studied the role of systemic biomarkers including IL-6 in 222 stable COPD patients and could not find a significant association between IL-6 and BODE index. Their failure to 
find a significant association might be related to the fact that they addressed age as a confounding factor in their study. The significant association of IL- 6 with BODE index and its components further supports the fact that a combination of systemic biomarkers along with BODE index assessment can be more accurate in predicting disease severity and progression. IL-6 is thought to be directly involved in the systemic inflammation and may be used as an additional parameter for risk assessment in patients with COPD.

\section{Clinical implications of the current findings}

Many systemic biomarkers predicting the clinical outcomes in terms of their relationship with mortality or exacerbations have recently been reported in COPD. Very few studies have looked into the predictive value of combining these biomarkers with multidimensional BODE index in assessing disease severity. We have shown an association between the expression of the serum biomarkers and the integrated systemic manifestations of the disease as represented by the functional capacity and the BODE index. The present analysis demonstrated that serum IL-6 concentration and BODE index combined can be a powerful prognostic determinant of the disease severity as well as its progression in patients with stable COPD. Of all the proinflammatory biomarkers studied, IL-6 is considered most accurately and significantly correlated with the maximum components of the BODE index.

\section{Strengths and limitations}

Our study has several strengths and limitations which merit consideration. To date, only a few studies have highlighted a relationship between disease severity measured by the BODE index and proinflammatory biomarkers in COPD patients. To the best of our knowledge, our study provides the largest hospital-based cross-sectional investigation evaluating the relationship of systemic inflammatory biomarkers with BODE index in a group of stable COPD patients and compares the results to those of healthy smokers. Then, we assessed a well-characterized, modest-sized monocentric cohort of COPD patients seeking treatment in the respiratory division of a premier tertiary academic institution of North India.

Our study also has some potential limitations. Firstly, this is a descriptive study, which depicts only associations; hence, we acknowledge that our analyses and conclusions will need to be replicated either prospectively in a study powered for these hypotheses or in other cohorts that contain similar data. Secondly, the biology of the inflammatory response is complex and we studied only a small panel of biomarkers. We did not study the markers of tissue repair, and it is likely that the balance between inflammation and repair is important for the pathobiology of COPD. Lastly, we had only few women in our study, so we cannot generalize the findings of our study to both sexes. Nevertheless, the findings of the current study need to be analyzed with a large sample size and a long-term follow-up period, involving different populations.

\section{Conclusion}

In summary, serum IL-6 combined with multidimensional BODE index appears to be an important, accurate biomarker for predicting disease severity in patients with stable COPD. In this setting, IL-6 is significantly correlated with the maximum components of BODE index as well as BODE index alone, compared to other biomarkers studied. Thus, we speculate that this novel biomarker may enable the determination of the severity and prediction of the course of the disease.

\section{Acknowledgments}

This study was supported by a grant from the Indian Council of Medical Research (ICMR), New Delhi. Dr Naushad Ahmad Khan is a recipient of a Scholarship Grant (Senior Research Fellowship) from ICMR. We presented an earlier version of the manuscript as an abstract at the American Thoracic Society 2015 International Conference, May 15-20, 2015 in Denver, Colorado, USA, as a poster presentation with interim findings.

The poster's abstract was published in "Poster Abstracts" in American Journal of Respiratory and Critical Care Medicine, Meeting Abstracts, American Thoracic Society 2015 International Conference, B38. Talking about COPD Biomarkers.

http://www.atsjournals.org/doi/abs/10.1164/ajrccmconference.2015.191.1 MeetingAbstracts.A2937 and http:// www.atsjournals.org/doi/book/10.1164/ajrccm-conference.2015.B38

\section{Author contributions}

NAK contributed substantially as the first author by designing the study, assisting in data collection, carrying out experimental work, interpreting the data, drafting the manuscript, and approving the final version of the manuscript. MKD contributed substantially as a coauthor by overall supervision of the project as the Principal Investigator, assisting in designing and conceptualization of the study, critically reviewing and revising the manuscript, and approving the final version of the manuscript. SK contributed substantially as a coauthor by reviewing all patient data, performing majority of statistical analyses and detailed review of the entire data, and revising the manuscript. IA contributed 
substantially as a coauthor by overseeing all data entry, helping in experimental and statistical analyses of the study, and revising the manuscript. GM contributed substantially as a coauthor by assisting in data collection, data entry, performing spirometry of the patients, and revising the manuscript. NK contributed substantially as a coauthor by designing the study, assisting in data collection, interpretation of the data and revising the manuscript critically and approving the final version of the manuscript. SAH contributed substantially as a senior author by designing the study, providing supervision of the project, revising the manuscript, and approving the final version of the manuscript.

\section{Disclosure}

The authors report no conflicts of interest in this work.

\section{References}

1. Qaseem A, Wilt TJ, Weinberger SE, et al. Diagnosis and management of stable chronic obstructive pulmonary disease: a clinical practice guideline update from the American College of Physicians, American College of Chest Physicians, American Thoracic Society, and European Respiratory Society. Ann Intern Med. 2011;155:179-191.

2. Mathers CD, Loncar D. Projections of global mortality and burden of disease from 2002 to 2030. PLoS Med. 2006;3(11):e442.

3. Seemungal TA, Hurst JR, Wedzicha JA. Exacerbation rate, health status and mortality in COPD --a review of potential interventions. Int J Chron Obstruct Pulmon Dis. 2009;4(1):203-223.

4. Nici L, Donner C, Wouters E, et al. American Thoracic Society/European Respiratory Society (ATS/ERS). Statement on Pulmonary Rehabilitation. Am J Respir Crit Care Med. 2006;173(12):1390-1413.

5. Celli BR, Cote CG, Marin JM, et al. The body-mass index, airflow obstruction, dyspnea, and exercise capacity index in chronic obstructive pulmonary disease. $N$ Engl J Med. 2004;350(4):1005-1012.

6. Global Initiative for Chronic Obstructive Lung Disease (GOLD), Global obstructive pulmonary disease. Available from: http://www.goldcopd. org/guidelines-global-strategy-for-diagnosismanagement. html. 2011. Accessed February 12, 2016.

7. Gan WQ, Man SF, Senthilselvan A, Sin DD. Association between chronic obstructive pulmonary disease and systemic inflammation: a systematic review and a meta-analysis. Thorax. 2004;59(7):574-580.

8. Chung KF. Inflammatory mediators in chronic obstructive pulmonary disease. Curr Drug Targets Inflamm Allergy. 2005;4(6):619-625.

9. de Torres JP, Cordoba-Lanus E, López-Aguilar C, et al. C-reactive protein levels and clinically important predictive outcomes in stable COPD patients. Eur Respir J 2006;27(5):902-907.

10. Pinto-Plata VM, Mullerova H, Toso JF, Feudjo-Tepie M, Soriano JB, Vessey RS, Celli BR. C-reactive protein in patients with COPD, control smokers and non-smokers. Thorax. 2006;61(1):23-28.

11. Schols AM, Buurman WA, Staal van den Brekel AJ, Dentener MA, Wouters EF. Evidence for a relation between metabolic derangements and increased levels of inflammatory mediators in a subgroup of patients with chronic obstructive pulmonary disease. Thorax. 1996;51(8): 819-824.

12. Broekhuizen R, Wouters EF, Creutzberg EC, Schols AM. Raised CRP levels mark metabolic and functional impairment in advanced COPD. Thorax. 2006;61(1):17-22.
13. de Godoy I, Donahoe M, Calhoun WJ, Mancino J, Rogers RM. Elevated TNF-alpha production by peripheral blood monocytes of weight-losing COPD patients. Am J Respir Crit Care Med. 1996;153(2):633-637.

14. Higashimoto $Y$, Yamagata $Y$, Taya $S$, et al. Systemic inflammation in chronic obstructive pulmonary disease and asthma: similarities and differences. Respirology. 2008;13(1):128-133.

15. Takabatake N, Nakamura H, Abe S, et al. The relationship between chronic hypoxemia and activation of the tumor necrosis factor-alpha system in patients with chronic obstructive pulmonary disease. Am J Respir Crit Care Med. 2000;161(4 Pt 1):1179-1184.

16. Yarnell JW, Sweetnam PM, Rumley A, Lowe GD. Lifestyle factors and coagulation activation markers: the Caerphilly Study. Blood Coagul Fibrinolysis. 2001;12(8):721-728.

17. ATS Committee on Proficiency Standards for Clinical Pulmonary Function Laboratories. ATS Statement: Guidelines for the six-minute walk test. Am J Respir Crit Care Med. 2002;166(1):111-117.

18. Mahler D, Wells CK. Evaluation of clinical methods for rating dyspnea. Chest. 1988;93(3):580-586.

19. Anthonisen NR, Wright EC, Hodgkin JE. Prognosis in chronic obstructive pulmonary disease. Am Rev Respir Dis. 1986;133(1):14-20.

20. Jones PW, Quirk FH, Baveystock CM, Littlejohns P. A selfcomplete measure of health status for chronic airflow limitation. The St George's Respiratory Questionnaire. Am Rev Respir Dis. 1992;145(6):1321-1327.

21. Ong KC, Earnest A, Lu SJ. A multidimensional grading system (BODE index) as predictor of hospitalization for COPD. Chest. 2005;128(6): 3810-3816.

22. Marin JM, Sanchez A, Alonso JE, et al. A multivariate grading system (BODE) as predictor of the severity of exacerbation in COPD (abstract). Am J Respir Crit Care Med 2003;167:A23.

23. Beeh KM, Beier J, Kornmann O, Mander A, Buhl R. Long-term repeatability of induced sputum cells and infl ammatory markers in stable, moderately severe COPD. Chest. 2003;123(3):778-783.

24. Balzano G, Stefanelli F, Iorio C, De Felice A, Melillo EM, Martucci M, Melillo G. Eosinophilic infl ammation in stable chronic obstructive pulmonary disease. Relationship with neutrophils and airway function. Am J Respir Crit Care Med. 1999;160(5 Pt 1):1486-1492.

25. Bon JM, Leader JK, Weissfeld JL, et al. The influence of radiographic phenotype and smoking status on peripheral blood biomarker patterns in chronic obstructive pulmonary disease.PLoS One 2009;4(8):e6865.

26. Tanni SE, Pelegrino NR, Angeleli AY, Correa C, Godoy I. Smoking status and tumor necrosis factor alpha mediated systemic inflammation in COPD patients. J Inflamm (Lond). 2010;7:29.

27. Di Francia M, Barbier D, Mege JL, Orehek J. Tumor necrosis factoralpha levels and weight loss in chronic obstructive pulmonary disease. Am J Respir Crit Care Med. 1994; 150(5 Pt 1):1453-1455.

28. Sarioglu N, Alpaydin AO, Coskun AS, Celik P, Ozyurt BC, YorganciogluA. Relationship between BODE index, quality of life and inflammatory cytokines in COPD patients. Multidiscip Respir Med. 2010;5(2):84-91.

29. Cosio MG, Saetta M, Agusti A. Immunologic aspects of chronic obstructive pulmonary disease. $N$ Engl J Med. 2009;360(23):2445-2454.

30. Aldonyte R, Jansson L, Piitulainen E, Janciauskiene S. Circulating monocytes from healthy individuals and COPD patients. Respir Res. 2003;4:11.

31. Pinto-Plata V, Casanova $C$, Müllerova $H$, et al. Inflammatory and repair serum biomarker pattern: association to clinical outcomes in COPD. Respir Res. 2012;13:71.

32. Eickhoff P, Valipour A, Kiss D, et al. Determinants of systemic vascular function in patients with stable chronic obstructive pulmonary disease. Am J Respir Crit Care Med. 2008;178(12):1211-1218.

33. Gaki E, Kontogianni K, Papaioannou AI, et al. Associations between BODE index and systemic inflammatory biomarkers in COPD. COPD. 2011;8(6):408-413. 


\section{Publish your work in this journal}

The Journal of Inflammation Research is an international, peer-reviewed open access journal that welcomes laboratory and clinical findings on the molecular basis, cell biology and pharmacology of inflammation including original research, reviews, symposium reports, hypothesis formation and commentaries on: acute/chronic inflammation; mediators of inflammation; cellular processes; molecular mechanisms; pharmacology and novel anti-inflammatory drugs; clinical conditions involving inflammation. The manuscript management system is completely online and includes a very quick and fair peer-review system. Visit http://www.dove press.com/testimonials.php to read real quotes from published authors.

Submit your manuscript here: https://www.dovepress.com/journal-of-inflammation-research-journal 